\title{
CONVERGENCE OF LINEAR APPROXIMATION OF ARCHIMEDEAN GENERATOR FROM WILLIAMSON'S TRANSFORM IN EXAMPLES
}

\author{
TOMÁŠ BACIGÁL — MÁRIA ŽDÍMALOVÁ
}

\begin{abstract}
We discuss a new construction method for obtaining additive generators of Archimedean copulas proposed by McNeil, A. J.-Nešlehová, J.: Multivariate Archimedean copulas, $d$-monotone functions and $l_{1}$-norm symmetric distributions, Ann. Statist. 37 (2009), 3059-3097, the so-called Williamson n-transform, and illustrate it by several examples. We show that due to the equivalence of convergences of positive distance functions, additive generators and copulas, we may approximate any $n$-dimensional Archimedean copula by an Archimedean copula generated by a transformation of weighted sum of Dirac functions concentrated in certain suitable points. Specifically, in two dimensional case this means that any Archimedean copula can be approximated by a piece-wise linear Archimedean copula, moreover the approximation of generator by linear splines circumvents the problem with the non-existence of explicit inverse.
\end{abstract}

\section{Introduction}

Copulas form an important class of multivariate dependence models. They have a lot of practical applications, including multivariate survival modelling. Recall that copulas aggregate 1-dimensional marginal distribution functions into $n$-dimensional $(n \geq 2)$ joint distribution functions. For more details we recommend [13].

We first define a copula. A function $C:[0,1]^{n} \rightarrow[0,1]$ is called a (n-dimensional) copula whenever it satisfies the boundary conditions (C1) and it is an $n$-increasing function, see $(\mathrm{C} 2)$, where:

(C) 2017 Mathematical Institute, Slovak Academy of Sciences.

2010 Mathematics Subject Classification: 60E05.

Keywords: Archimedean copula, Williamson, approximation.

The work on this paper was supported by grant APVV-14-0013 and VEGA 1/0420/15. 


\section{TOMÁŠ BACIGÁL — MÁRIA ŽDÍMALOVÁ}

(C1) $C\left(x_{1}, \ldots, x_{n}\right)=0$ whenever $0 \in\left\{x_{1}, \ldots, x_{n}\right\}$, i.e., 0 is an annihilator of $\mathrm{C}$, and $C\left(x_{1}, \ldots, x_{n}\right)=x_{i}$ whenever $x_{j}=1$ for each $j \neq i$ (i.e., 1 is a neutral element of $C$ ).

(C2) For any $\mathbf{x}, \mathbf{y} \in[0,1]^{n}, \mathbf{x} \leq \mathbf{y}$, it holds

$$
V_{C}([\mathbf{x}, \mathbf{y}])=\sum_{\varepsilon \in\{-1,1\}^{n}}\left(C\left(\mathbf{z}_{\varepsilon}\right) \prod_{i=1}^{n} \varepsilon_{i}\right) \geq 0,
$$

where $\mathbf{z}_{\varepsilon}=\left(z_{1}^{\varepsilon_{1}}, \ldots, z_{n}^{\varepsilon_{n}}\right), z_{i}^{1}=y_{i}, z_{i}^{-1}=x_{i}$.

Note that $V_{C}([\mathbf{x}, \mathbf{y}])$ is called the $C$-volume of the $n$-dimensional interval $(n$-box $)$ $[\mathbf{x}, \mathbf{y}]$.

Due to Skla r's theorem [16] for a random vector $Z=\left(X_{1}, \ldots, X_{n}\right)$, a function $F_{Z}: R^{n} \rightarrow[0,1]$ is a joint distribution function of $Z$ if and only if there is a copula $C:[0,1]^{n} \rightarrow[0,1]$ so that

$$
F_{Z}\left(x_{1}, \ldots, x_{n}\right)=C\left(F_{X_{1}}\left(x_{1}\right), \ldots, F_{X_{n}}\left(x_{n}\right)\right),
$$

where $F_{X_{i}}: R \rightarrow[0,1]$ is a distribution function related to the random variable $X_{i}, i=1, \ldots, n$. The copula $C$ in (11) is unique whenever random variables $X_{1}, \ldots, X_{n}$ are continuous. For some other details on copulas see [5] and [13].

Hereafter we will consider a class of copulas named Archimedean copulas. In the simplest case, Archimedean 2-copulas are characterized by the associativity of $C$ and the diagonal inequality $C(x, x)<x$ for all $x \in] 0,1[$. They are necessarily symmetric, i.e., they can model the stochastic dependence of exchangeable random variables $(X, Y)$ only, yet their popularity in practice (hydrology, financial, and other applied areas) is indisputable, mainly due to the representation using one-dimensional functions called generators as shown in the next result, attributed to Moynihan [12].

TheOrem 1. A function $C:[0,1]^{2} \rightarrow[0,1]$ is an Archimedean copula if and only if there is a convex (i.e., a 2-monotone) continuous strictly decreasing function $f:[0,1] \rightarrow[0, \infty], f(1)=0$, so that

$$
C(x, y)=f^{(-1)}(f(x)+f(y)),
$$

where the pseudo-inverse $f^{(-1)}:[0, \infty] \rightarrow[0,1]$ is given by

$$
f^{(-1)}(u)=f^{-1}(\min (u, f(0))) .
$$

The function $f$ is called an additive generator of the copula $C$, and it is unique up to a positive multiplicative constant.

Let $\mathcal{F}_{2}$ be the class of all additive generators of binary copulas characterized in the above theorem. More details about the generators can be found in [5], [6], [13] and about construction methods for additive generators in [1, [2], 4], [7], 11]. 
Before we review several known facts for additive generators of copulas, let us briefly recall a link between copula $C$ and Spearman's correlation coefficient $\rho$,

$$
\rho=12 E[U V]-3=12 \iint_{[0,1]^{2}} u v d C(u, v)-3=12 \iint_{[0,1]^{2}} C(u, v) d u d v-3
$$

as well as Kendall's correlation coefficient $\tau$,

$$
\tau=4 E[C(U, V)]-1=4 \iint_{[0,1]^{2}} C(u, v) d C(u, v)-1,
$$

where $U=F_{X}(X)$ and $V=F_{Y}(Y)$ are uniformly distributed random variables that are connected by the same copula as are $X$ and $Y$. Alternatively, Kendall's tau can be computed directly from copula generator,

$$
\tau=1+4 \int_{0}^{1} \frac{f(t)}{f^{\prime}(t)} d t=1-4 \int_{0}^{\infty} t\left(f^{(-1)^{\prime}}(t)\right)^{2} d t
$$

which is far more convenient.

Any binary Archimedean copula $C:[0,1]^{2} \rightarrow[0,1]$ generated by an additive generator $f:[0,1] \rightarrow[0, \infty]$, is also a triangular norm [6], [15] and thus, it can be univocally extended to an $n$-ary function (we keep the original notation also for this extension) $C:[0,1]^{n} \rightarrow[0,1]$ given by

$$
C\left(x_{1}, \ldots, x_{n}\right)=f^{(-1)}\left(\sum_{i=1}^{n} f\left(x_{i}\right)\right) .
$$

Obviously, for any $n \geq 2, C$ satisfies the boundary conditions (C1). However, for $n>2$, (C2) may fail. For example, the smallest binary copula $W:[0,1]^{2} \rightarrow[0,1]$ given by $W(x, y)=\max (0, x+y-1)$ is generated by the additive generator $f_{W}:[0,1] \rightarrow[0, \infty], f_{W}(x)=1-x$. Its $n$-ary extension is given by

$$
W\left(x_{1}, \ldots, x_{n}\right)=1-\min \left(1, \sum_{i=1}^{n}\left(1-x_{i}\right)\right)=\max \left(0, \sum_{i=1}^{n} x_{i}-(n-1)\right) .
$$

Consider $\mathbf{x}, \mathbf{y} \in[0,1]^{n}, \mathbf{x}=\left(\frac{1}{2}, \ldots, \frac{1}{2}\right), \mathbf{y}=(1, \ldots, 1)$. Then $V_{W}([\mathbf{x}, \mathbf{y}])=1-\frac{n}{2}$, i.e., this volume is negative whenever $n>2$, which shows that $W$ is a copula only for $n=2$. A complete description of additive generators of binary copulas such that the corresponding generated $n$-ary function is also an $n$-ary copula, $n>2$, was given by McNeil and Nešlehová in [8] and is recalled in the next theorem.

Theorem 2. Let $f:[0,1] \rightarrow[0, \infty]$ be a continuous strictly decreasing function such that $f(1)=0$ (i.e., $f$ is an additive generator of a continuous Archimedean $t$-norm, see [6]). Then the $n$-ary function $C:[0,1]^{n} \rightarrow[0,1]$ given by (5) 


\section{TOMÁŠ BACIGÁL — MÁRIA ŽDÍMALOVÁ}

is an $n$-ary copula if and only if the function $g:[-\infty, 0] \rightarrow[0,1]$ given by $g(u)=f^{(-1)}(-u)$ is $(n-2)$-times differentiable with non-negative derivatives $g^{\prime}, \ldots, g^{(n-2)}$ on $]-\infty, 0\left[\right.$ (or equivalently, $(-1)^{n}\left(f^{(-1)}\right)^{(n)}(u) \geq 0$ ), and $g^{(n-2)}$ is a convex function (see Figure 1).

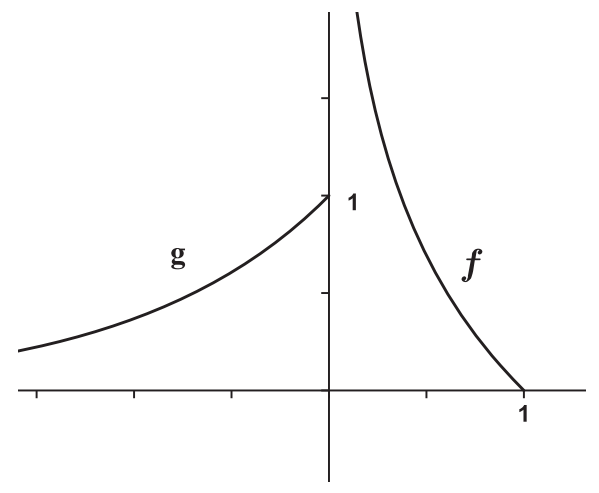

FiguRE 1. Illustration of a generator $f$ and its corresponding function $g$.

We denote by $\mathcal{F}_{n}$ the class of all additive generators that generate $n$-ary copulas as characterized in Theorem 2.

Additive generators, which generate an $n$-ary copula for any $n \geq 2$, are called universal generators. Due to Theorem 2 , we have the next result, see also 8 .

Corollary 1. Let $f:[0,1] \rightarrow[0, \infty]$ be an additive generator of a binary copula $C:[0,1]^{2} \rightarrow[0,1]$. Then the $n$-ary extension $C:[0,1]^{n} \rightarrow[0,1]$ given by (5) is an $n$-ary copula for each $n \geq 2$ if and only if the function $g:[-\infty, 0] \rightarrow[0,1]$ given by $g(u)=f^{(-1)}(-u)$ is absolutely monotone, i.e., $g^{(k)}$ exists and is non-negative for each $k \in N=\{1,2, \ldots\}$.

The class of all universal additive generators will be denoted by $\mathcal{F}_{\infty}$. It is not difficult to check that $\mathcal{F}_{2} \supset \mathcal{F}_{3} \supset \cdots \supset \mathcal{F}_{\infty}$.

The reverse problem of characterization of $n$-ary copulas which are generated by an additive generator was solved by $\mathrm{S}$ t u p ň a n o vá and K o l e s á r o vá [17.

Theorem 3. Let $C:[0,1]^{n} \rightarrow[0,1]$ be an n-ary copula, $n>2$. Then $C$ is generated by an additive generator $f:[0,1] \rightarrow[0, \infty]$ if and only if $C$ satisfies the diagonal inequality $C(x, \ldots, x)<x$ for all $x \in] 0,1[$, and $C$ is associative in the Post sense, i.e., for any $x_{1}, \ldots, x_{2 n-1} \in[0,1]$ it holds

$$
\begin{aligned}
C\left(C\left(x_{1}, \ldots, x_{n}\right), x_{n+1}, \ldots, x_{2 n-1}\right) & = \\
=C\left(x_{1}, C\left(x_{2}, \ldots, x_{n+1}\right), x_{n+2}, \ldots, x_{2 n-1}\right) & =\ldots \\
\ldots & =C\left(x_{1}, \ldots, x_{n-1}, C\left(x_{n}, \ldots, x_{2 n-1}\right)\right) .
\end{aligned}
$$




\section{CONVERGENCE OF LINEAR APPROXIMATION OF ARCHIMEDEAN GENERATOR}

The $n$-monotone Archimedean copula generators may be characterized using a little known integral transform introduced by W ill i a m s o n in 1956, see [18. In Mc Neil and Nešlehová [8] there is a description of this transform, which, for a fixed $n \geq 2$, will be called the Williamson $n$-transform. In what follows, we discuss the Williamson $n$-transform and illustrate it by examples.

\section{The Williamson $n$-transform}

An interesting link between additive generators of copulas and positive distance functions [9], i.e., distribution functions with support in ]0, $\infty[$, was described in details in [8]. Based on the results of Williamson [18, we recall the next important result.

Theorem 4 (McNeil and Nešlehová [8], Corollary 3.1). The following claims are equivalent for an arbitrary $n \in\{2,3, \ldots\}$ :

(i) $f \in \mathcal{F}_{n}$.

(ii) Under the notation of Theorem 2 , the function $F$ : $]-\infty, \infty[\rightarrow[0,1]$ given by $F(x)=0$ if $x \leq 0$, and for $x>0$,

$$
F(x)=1-\sum_{k=0}^{n-2} \frac{(-1)^{k} x^{k}\left(f^{(-1)}\right)^{(k)}(x)}{k !}-\frac{(-1)^{n-1} x^{n-1}\left(f^{(-1)}\right)_{+}^{(n-1)}(x)}{(n-1) !}
$$

is a distribution function of a positive random variable $X$ (i.e., $P(X \leq 0)=0$ ), where $\cdot_{+}^{(n-1)}$ denotes the right-derivative of order $n-1$.

Note that due to [18, if $F$ is a positive distance function, i.e., a distribution function of a positive random variable $X$, then for a fixed $n \in\{2,3, \ldots\}$ the Williamson $n$-transform provides an inverse transformation to (6),

$$
\begin{array}{r}
f^{(-1)}(x)=\int_{x}^{\infty}\left(1-\frac{x}{t}\right)^{n-1} d F(t)= \begin{cases}\max \left(0, E\left[1-\frac{x}{X}\right]^{n-1}\right), & x>0 \\
1-F(0), & x=0\end{cases} \\
\text { where } \quad x \in\left[0, \infty\left[\text { and } f^{(-1)}(\infty)=0 .\right.\right.
\end{array}
$$

Note that a similar relationship can be shown between additive generators from $\mathcal{F}_{\infty}$ and positive distance functions, based on the Laplace transform, i.e,

$$
f^{(-1)}(x)=\int_{0}^{\infty} e^{-x t} d F(t)
$$

For more and interesting details we recommend [8]. 


\section{TOMÁŠ BACIGÁL — MÁRIA ŽDÍMALOVÁ}

Let $F$ be a distance function related to a positive random variable $X$. For any $c>0$, the random variable $c . X$ possesses the distance function $F_{c}$ given by $F_{c}(x)=F\left(\frac{x}{c}\right)$. Then, for any $n \in\{2,3, \ldots\}$,

$$
\begin{aligned}
f_{c}^{(-1)}(x) & =\int_{x}^{\infty}\left(1-\frac{x}{t}\right)^{n-1} d F_{c}(t) \\
& =\int_{x}^{\infty}\left(1-\frac{x}{t}\right)^{n-1} d F\left(\frac{t}{c}\right) \\
& =\int_{\frac{x}{c}}^{\infty}\left(1-\frac{x}{c u}\right)^{n-1} d F(u) \\
& =f^{(-1)}\left(\frac{x}{c}\right) .
\end{aligned}
$$

Obviously, for the related additive generators it holds that $f_{c}=c$. $f$, i.e., they generate the same copula. Vice versa, clearly from (6) it follows that if two generators generate the same ( $n$-ary) Archimedean copula, the corresponding positive random variables differ only in a positive multiplicative constant. The next result follows.

TheORem 5. For each $n \in\{2,3, \ldots\}$, there is an one-to-one correspondence between the class $\mathcal{F}_{n}$ and the class $\mathcal{H}$ of all factor classes of positive distance functions related to the equivalence $F \sim G$ if and only if $G(x)=F\left(\frac{x}{c}\right)$ for some $c>0$.

In the following, we illustrate the construction method by few examples.

ExAmple 1. Let $F$ be equal to a Dirac function 1 focused at point $x_{0}=1$,

$$
F(x)=\delta_{1}(x)= \begin{cases}0, & x<1 \\ 1, & 1 \leq x\end{cases}
$$

then, as it is also shown in [8], by the Williamson $n$-transform we get generator $f_{n}(x)=1-x^{\frac{1}{n-1}}$ of the weakest $n$-dimensional Archimedean copula, i.e., the non-strict Clayton copula with parameter $\lambda=\frac{-1}{n-1}$, see Figure 2. By rescaling generator to $\tilde{f}_{n}(x)=\frac{f(x)}{f(1 / 2)}, x \in[0,1]$, the copula would not change, yet such a generator is fixed to the value $\tilde{f}_{n}\left(\frac{1}{2}\right)=1$, which we will use later to show convergence.

${ }^{1}$ Dirac function is defined as $\delta_{x_{0}}(x)= \begin{cases}0, & x<x_{0}, \\ 1, & x \geq x_{0} .\end{cases}$ 


\section{CONVERGENCE OF LINEAR APPROXIMATION OF ARCHIMEDEAN GENERATOR}
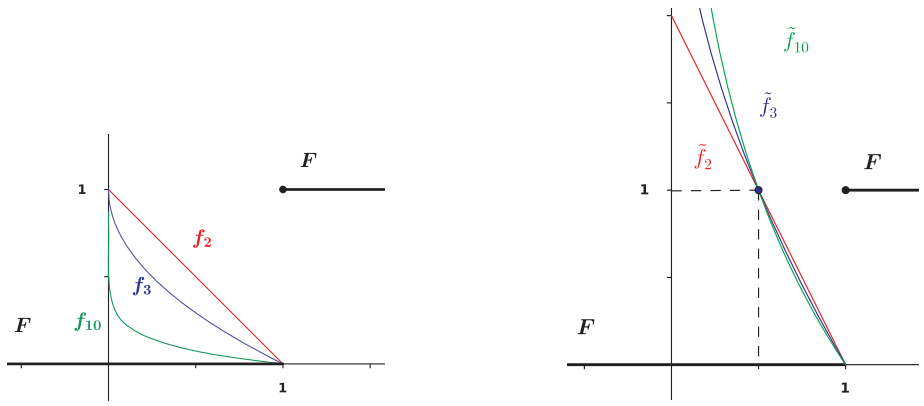

FiguRE 2. Dirac function $F$, the corresponding generators $f_{n}$ for different $n$ and rescaled generators $\tilde{f}_{n}$.

EXAMPLE 2. Let $F$ be a uniform probability distribution function

$$
F(x)=\left\{\begin{array}{ll}
0, & x<a \\
\frac{x-a}{b-a}, & a \leq x<b \\
1, & b \leq x .
\end{array} \quad \text { with } 0 \leq a<b,\right.
$$

Then for dimension $n=2$ we get

$$
\begin{aligned}
f_{2}^{(-1)}(x) & =\int_{x}^{\infty}\left(1-\frac{x}{t}\right)^{2-1} F^{\prime}(t) d t \\
& = \begin{cases}\int_{a}^{b}\left(1-\frac{x}{t}\right) \frac{1}{b-a} d t, & x<a, \\
\int_{x}^{b}\left(1-\frac{x}{t}\right) \frac{1}{b-a} d t, & a \leq x<b, \\
\int_{x}^{\infty}\left(1-\frac{x}{t}\right) 0 d t, & b \leq x,\end{cases} \\
& = \begin{cases}\frac{1}{b-a}[t-x \log t]_{a}^{b}=1-\frac{x \log \left(\frac{b}{a}\right)}{b-a}, & x<a, \\
\frac{1}{b-a}[t-x \log t]_{x}^{b}=\frac{b}{b-a}-\frac{x+x \log \left(\frac{b}{x}\right)}{b-a}, & a \leq x<b, \\
0, & b \leq x\end{cases}
\end{aligned}
$$

(where $F^{\prime}$ denotes a first derivative of $F$ ) from which the corresponding generator can be obtained only numerically, and so is the case also with the higher dimensions, e.g.,

$$
f_{3}^{(-1)}(x)= \begin{cases}1-\frac{2 x \log \left(\frac{b}{a}\right)}{b-a}+\frac{x^{2}}{a b}, & x<a, \\ \frac{b}{b-a}-2 x \log \left(\frac{b}{x}\right)-\frac{x^{2}}{(b-a) b}, & a \leq x<b, \\ 0, & b \leq x,\end{cases}
$$

displayed in Figure 3. Setting $a=0$ we get $\tau=0$ regardless of parameter $b$, which is in clear accordance with Theorem 5 , 


\section{TOMÁŠ BACIGÁL — MÁRIA ŽDÍMALOVÁ}

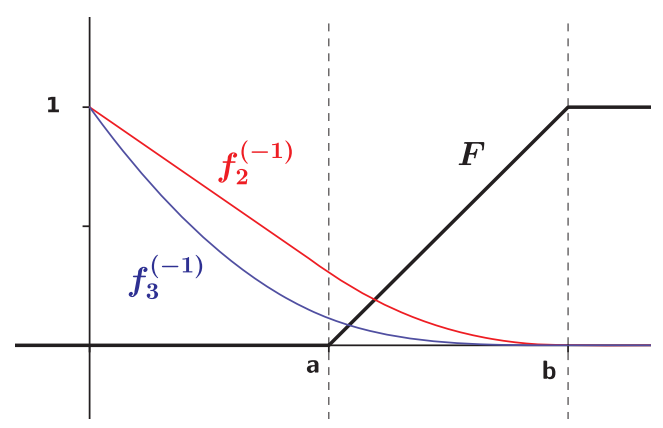

FIgURE 3. Uniform $\mathrm{U}(\mathrm{a}, \mathrm{b})$ probability distribution function $F$ and pseudoinverses of the corresponding generators $f_{n}$.

We continue with the examples of constructing generators of non-strict Archimedean copulas while restricting the support of univariate distribution in the unit interval. By applying a suitable increasing transformation (such as power function) to a positive distance function on $[0,1]$ we obtain a new distribution.

Example 3. Consider a positive distance function $F(x)=\min \left(1, x^{2}\right)$ and the corresponding density $F^{\prime}(x)=2 x$ on $[0,1]$. Then

$$
\begin{aligned}
f_{2}^{(-1)}(x) & =\int_{x}^{\infty}\left(1-\frac{x}{t}\right)^{2-1} d F(t) \\
& = \begin{cases}\int_{x}^{1}(t-x) \frac{2 t}{t} d t=(1-x)^{2}, & 0 \leq x \leq 1, \\
0, & 1<x\end{cases} \\
& =\max (1-x, 0)^{2} .
\end{aligned}
$$

Then the generator $f_{2}(x)=1-\sqrt{x}, x \in[0,1]$, is the generator of Clayton copula for parameter $\lambda=-\frac{1}{2}$. Nevertheless, in higher dimensions, $n \geq 3$, the generator has no closed form, e.g., $f_{3}^{(-1)}(x)=1-4 x+x^{2}(3-2 \log x)$ for $x \in[0,1]$ and 0 , otherwise (see Figure 4).

ExAmple 4. Let us generalize Example 3 and start with a parametric family $F(x)=\min \left(1, x^{p}\right)$, where $p>0$. Observe that $\lim _{x \rightarrow 0} F(x)=\delta_{0}(x)$ while $\lim _{x \rightarrow \infty} F(x)=\delta_{1}(x)$. Then

$$
f_{2}^{(-1)}(x)= \begin{cases}\frac{x^{p}-p x+p-1}{p-1}, & 0 \leq x \leq 1 \wedge p \neq 1 \\ x(\log x-1)+1, & 0 \leq x \leq 1 \wedge p=1 \\ 0, & 1<x\end{cases}
$$




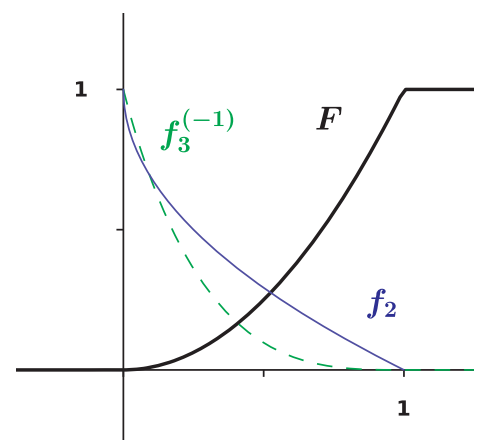

Figure 4. Illustration of Example 3 with non-invertible case $n=3$.

Though it lacks an explicit inverse, the copulas that it generates cover almost whole dependence range with $\tau=1-\frac{2 p}{1+p}, \tau \in(0,1)$, and we will use it later to demonstrate approximation approach. Figure 5 shows simulations from this parametric copula family for $p=0.5$ and $p=2$. The only tail dependence is present at the upper tail for $p \in(0,1)$.

It is interesting to illustrate also the inverse Williamson $n$-transform.
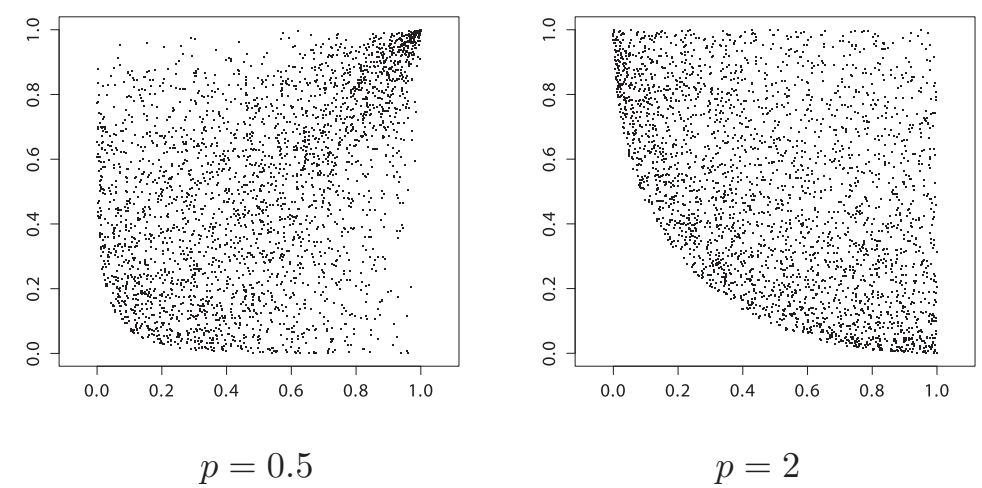

FiguRE 5. Sampling from copula family constructed in Example 4

EXAMPLE 5. Let us take a generator of:

- the Ali-Mikhail-Haq copula $f(x)=\frac{1}{x}-1$ corresponding to the parameter $\lambda=1$ and denote by $F_{n}, n=2,3, \ldots$, a positive distance function related to $f$ through (6). Then $F_{n}(x)=1-\frac{1}{1+x}-\frac{x}{(1+x)^{2}}-\cdots-\frac{x^{n-1}}{(1+x)^{n}}=\left(\frac{x}{1+x}\right)^{n}$ which can be viewed as a parametric subfamily of all positive valued distribution functions $F_{p}$ with any positive parameter $p$; 


\section{TOMÁŠ BACIGÁL — MÁRIA ŽDÍMALOVÁ}

- the product copula $f(x)=-\frac{1}{p} \log x$ with constant $p>0$ and inverse $f^{-1}(x)=$ $\exp (-p x)$. From (6) for $n=2$ we get $F(x)=1-\exp (-p x)(1-p x)$. By comparing the density $\frac{\partial F(x)}{\partial x}=p^{2} x \exp (-p x)$ and the convolution of two exponential distribution $\mathcal{D}_{\lambda}$ densities with parameter $\lambda>0$,

$$
\int_{0}^{x} \lambda \exp (-\lambda t) \lambda \exp (-\lambda(x-t)) d t=\lambda^{2} x \exp (-\lambda x)
$$

it becomes clear that the resulting distribution is a distribution of the random variable $Y=X_{1}+X_{2}$, where $X_{1}, X_{2} \sim \mathcal{D}_{\lambda}$ are independent (and identically distributed) random variables. The relation holds for any $n \geq 2$, thus (6) yields a cumulative distribution function of the sum of i.i.d. random variables $X_{1}, \ldots, X_{n} \sim \mathcal{D}_{p}, F_{X_{1}+\cdots+X_{n}}(x)=1-\exp (-p x) \sum_{i=1}^{n} \frac{(p x)^{i-1}}{(i-1) !}$ with $p>0$ which defines the Erlang distribution with rate parameter $p$ and shape parameter $n$.

To complete the examples, let us illustrate also the Laplace transform.

EXAMPLE 6. Starting with positive distance function of:

- discrete random variable with probability mass concentrated in $\lambda>0$, i.e., Dirac function $F(x)=0$ for $x<\lambda$ and 1 otherwise, then the Laplace transform leads through $g(x)=\exp (\lambda x)$ to the product copula $\Pi$.

- exponential distribution $F(x)=1-\exp (-\lambda x), \lambda>0$, we get $f^{-1}(x)=$ $\left(\frac{\lambda}{x+\lambda}\right)$ and $f(x)=\lambda\left(\frac{1}{x}-1\right)$ which generates the same copula (Clayton copula with parameter equal to 1) regardless of the choice of $\lambda$.

Now we focus on the Dirac function since it can be viewed as a building block for distribution functions of a random variable with probability mass concentrated in $l$ discrete points. Immediately a question arises: if such a distribution functions can approximate distribution of a continuous r.v. (for any $l$, going possibly to infinity), does this convergence imply also a convergence of the corresponding generators and even a convergence of the generated copulas?

\section{Convergence theorems}

Definition 1. Let $\left(F_{m}\right)_{m}$ be a sequence of distribution functions and let $F$ be a distribution function. We say that the sequence of distribution functions $F_{m}$, $m=1,2, \ldots$, weakly converge to distribution function $F$ if

$$
\lim _{m \rightarrow \infty} F_{m}(x)=F(x)
$$

holds for any point $x \in R$ in which $F$ is continuous. The weak convergence will be denoted by $F_{m} \stackrel{w}{\rightarrow} F$. 


\section{CONVERGENCE OF LINEAR APPROXIMATION OF ARCHIMEDEAN GENERATOR}

Recall that the Lévy-Cramér continuity theorem 14 ensures the convergence $\int_{-\infty}^{\infty} h(t) d F_{m}(t) \underset{m \rightarrow \infty}{\longrightarrow} \int_{-\infty}^{\infty} h(t) d F(t)$, where $\left.h:\right]-\infty, \infty[\rightarrow]-\infty, \infty[$ is a continuous bounded real function and $F_{m} w \rightarrow F$. Obviously, for any $n \geq 2$ and $x<0$, the function $h:]-\infty, \infty[\rightarrow]-\infty, \infty\left[\right.$ given by $h(t)=\left(1+\frac{x}{t}\right)^{n-1} \delta_{-x}(t)$ is continuous and bounded. This fact proves the next important result.

TheOREM 6. Let a sequence $\left(F_{m}\right)_{m}$ of distance functions converge weakly to a distance function $F, F_{m} \stackrel{w}{\rightarrow} F$. Then, for any $n \geq 2$, the corresponding additive generators $f, f_{m}, m=1,2, \ldots$, of $n$-dimensional Archimedean copulas are related by the pointwise convergence $f_{m} \underset{m \rightarrow \infty}{\longrightarrow} f$.

P r o o f. Based on the Williamson $n$-transform (77) and the Lévy-Cramér continuity theorem, $g_{m} \rightarrow g$ pointwisely, and all functions $g, g_{m}, m=1,2, \ldots$ are convex, continuous and strictly increasing. Then also the related additive generators $f, f_{m}, m=1,2, \ldots$, satisfy $f_{m} \rightarrow f$ pointwisely.

The reverse of Theorem 6 is based on the next lemma.

Lemma 1. Let $\left(f_{m}\right)_{m}, f$ be convex real functions defined on a real interval $] \alpha, \beta[$ such that $f_{m} \rightarrow f$ pointwisely. Then for any point $\left.a \in\right] \alpha, \beta\left[\right.$, where $f^{\prime}(a)$ exists it holds $\lim _{m \rightarrow \infty} f^{\prime}\left(a^{-}\right)=f^{\prime}(a)=\lim _{m \rightarrow \infty} f_{m}^{\prime}\left(a^{+}\right)$.

Proof. Note that due to the convexity, the left derivatives $f_{m}^{\prime}\left(a^{-}\right), f_{m}^{\prime \prime}\left(a^{-}\right)$ and the right derivatives $f_{m}^{\prime}\left(a^{+}\right), f_{m}^{\prime \prime}\left(a^{+}\right)$exist at each point $\left.a \in\right] \alpha, \beta[$. Moreover, the convexity ensures also that $f_{m}(x) \geq f_{m}(a)+(x-a) f_{m}^{\prime}\left(a^{-}\right)$and $f_{m}(x) \geq f_{m}(a)+(x-a) f_{m}^{\prime}\left(a^{+}\right)$for any $m=1,2, \ldots$ and $\left.x \in\right] \alpha, \beta[$. Fix $a \in] \alpha, \beta\left[\right.$. Then, for any $x>a, f_{m}^{\prime}\left(a^{-}\right) \leq \frac{f_{m}(x)-f_{m}(a)}{x-a}$ and thus $\lim \sup f_{m}^{\prime}\left(a^{-}\right) \leq$ $\frac{f(x)-f(a)}{x-a}$. Therefore $\limsup f_{m}^{\prime}\left(a^{-}\right) \leq \lim _{x \rightarrow a^{+}} \frac{f(x)-f(a)}{x-a}=f^{\prime}\left(a^{+}\right)$. Similarly, $\lim \inf f_{m}^{\prime}\left(a^{-}\right) \geq f^{\prime}\left(a^{-}\right)$, which implies the existence of the limit of $\left(f_{m}^{\prime}\left(a^{-}\right)\right)_{m}$, whenever $f^{\prime}\left(a^{-}\right)=f^{\prime}\left(a^{+}\right)=f^{\prime}(a), \lim _{m \rightarrow \infty} f_{m}^{\prime}\left(a^{-}\right)=f^{\prime}(a)$ if $f^{\prime}(a)$ exists. Using similar arguments, if $f^{\prime}(a)$ exists, then also $\lim _{m \rightarrow \infty} f_{m}^{\prime}\left(a^{+}\right)=f^{\prime}(a)$.

Based on Lemma 1, the next result follows directly.

TheOREM 7. Let $f_{m}, f \in \mathcal{F}_{n}, m=1,2, \ldots$, be additive generators of $n$-dimensional Archimedean copulas, such that $f_{n} \rightarrow f$ pointwisely on $\left.] 0,1\right]$. Let $F_{m}, F$, $m=1,2, \ldots$, be the related distance function obtained by means of the transform ([6). Then $F_{m} \stackrel{w}{\rightarrow} F$.

Pr o of. Based on Theorem 2, $g_{m}^{(k)}, m=1,2, \ldots$, and $g^{(k)}$ are convex functions for $k=0,1, \ldots, n-2$. The pointwise convergence $f_{m} \rightarrow f$ on $\left.] 0,1\right]$ implies the pointwise convergence $g_{m} \rightarrow g$ on $]-\infty, 0[$, and due to Lemma[1] repeated $(n-2)$ -times, it holds $\lim _{m \rightarrow \infty} g_{m}^{\prime}(x)=g^{\prime}(x), \ldots, \lim _{m \rightarrow \infty} g_{m}^{(n-2)}(x)=g^{(n-2)}(x)$ and 
$\lim _{m \rightarrow \infty} g_{m}^{(n-1)}\left(x^{-}\right)=g^{(n-1)}(x)$ at each point $\left.x \in\right]-\infty, 0\left[\right.$, where $g^{(n-1)}(x)$ exists. Therefore, $\lim _{m \rightarrow \infty} F_{m}(x)=F(x)$ at each point $\left.x \in\right] 0, \infty$ [, where the function $g^{(n-1)}\left(x^{-}\right)$is continuous, i.e., where $F(x)$ is continuous. Thus $F_{m} w \rightarrow F$.

The next result from [10] was shown for ( $n$-ary) continuous Archimedean triangular norms (however, any ( $n$-ary) Archimedean copula is also a continuous Archimedean t-norm) and later for Archimedean copulas in [3, Proposition 2].

Theorem 8. Let $C, C_{m}:[0,1]^{n} \rightarrow[0,1], m=1,2, \ldots$, be continuous Archimedean copulas, generated by additive generators $f, f_{m}:[0,1] \rightarrow[0, \infty], m=1,2, \ldots$, respectively. Then the following are equivalent.

i) $C_{m} \underset{m \rightarrow \infty}{\longrightarrow} C$ pointwisely.

ii) There are positive constants $c_{m}, m=1,2, \ldots$, so that $c_{m} f_{m} \rightarrow f$ pointwisely.

Combining Theorems 6, 7, 8 we have the next result which can be exploited when approximating Archimedean copulas.

COROLlARY 2. The following convergences of related objects are equivalent (for any $n \geq 2$ ):

i) for distance functions, $F_{m} \stackrel{w}{\rightarrow} F$;

ii) for additive generators from $\mathcal{F}_{n}, f_{m} \rightarrow f$ pointwisely on $\left.] 0,1\right]$;

iii) for $n$-dimensional Archimedean copulas, $C_{m} \rightarrow C$ pointwisely.

Recall that each distance function $F$ can be obtained as a weak limit of (bounded) discrete distance functions $F_{m}$, and that each bounded discrete distance function is, in fact, a convex combination of Dirac distance functions.

\section{Approximation}

In this section we are interested mainly in $(n=2)$-dimensional case, since it is of most benefit in practice. Therefore hereafter the subscript with generator $f$ gains a different meaning: the number of pieces $f$ is approximated by.

ExAmple 7 . Let $F(x)=\min \left(1, x^{2}\right)$ be the positive distance function from the Example 3 and function

$$
F_{2}(x)=F\left(\frac{1}{2}\right) \delta_{\frac{1}{2}}(x)+\left(F(1)-F\left(\frac{1}{2}\right)\right) \delta_{1}(x)= \begin{cases}0, & x<\frac{1}{2} \\ \frac{1}{4}, & \frac{1}{2} \leq x<1 \\ 1, & 1 \leq x\end{cases}
$$


approximates $F$ by means of a sum of $m=2$ Dirac functions concentrated in respective points $\left(\frac{1}{2}, \frac{1}{4}\right),\left(1, \frac{3}{4}\right)$. Then the Williamson transform with $n=2$ yields

$$
f_{2}^{(-1)}(x)=\frac{1}{4} \max \left(0,1-\frac{x}{\frac{1}{2}}\right)+\frac{3}{4} \max \left(0,1-\frac{x}{1}\right)= \begin{cases}1-\frac{5}{4} x, & x<\frac{1}{2} \\ \frac{3}{4}-\frac{3}{4} x, & \frac{1}{2} \leq x<1 \\ 0, & 1 \leq x\end{cases}
$$

From Example 7 illustrated in Figure 6 we see that for $n=2$ the additive generator inverse $f_{2}^{(-1)}$ is piecewise linear and does not coincide with $f^{(-1)}$ in the interval $] 0,1[$.

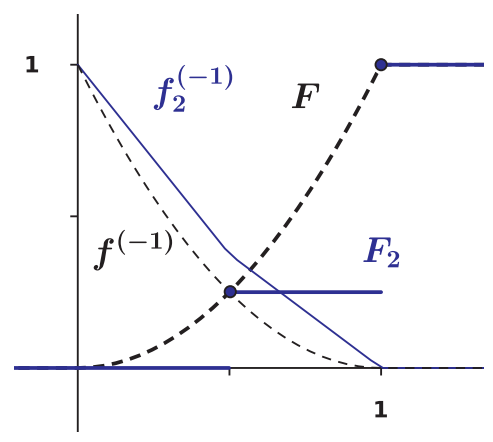

FiguRE 6. Approximation by the sum of $m=2$ Dirac functions.

Dividing an interval $\left[a_{0}, a_{m}\right]$ by points $\left\{a_{i}\right\}_{i=1, \ldots m}, a_{0}<a_{1}<\cdots a_{m}$, with concentration of probability given by some probability mass function $p(x)$, the approximate positive distance function

$$
F_{m}(x)=\sum_{i=1}^{m} p\left(a_{i}\right) \delta_{a_{i}}(x)
$$

is then transformed by (7) to the generator inverse (related to some $n$-dimensional Archimedean copula)

$$
f_{m}^{(-1)}(x)=\sum_{x<a_{i}} p\left(a_{i}\right)\left(1-\frac{x}{a_{i}}\right)^{n-1}=\sum_{i=1}^{m} p\left(a_{i}\right) \max \left(0,1-\frac{x}{a_{i}}\right)^{n-1} .
$$

Observe that the function $f_{m}^{(-1)}$ in Eq. (9) is an $(n-1)$-dimensional spline. For $n=2$, both $f_{m}^{(-1)}$ and the corresponding additive generator $f_{m}$ are linear splines, and the related Archimedean copula $C_{m}$ is piece-wise linear, as shown in Example 10. In the opposite direction, denote $b_{i}=f_{m}^{(-1)}\left(a_{i}\right)$ and $p_{i}=p\left(a_{i}\right)$ for $i=1,2 \ldots m$ with $b_{0}=1$ corresponding to $a_{0}=0$ and, clearly, $b_{m}=0$. 


\section{TOMÁŠ BACIGÁL — MÁRIA ŽDÍMALOVÁ}

Having points $\left\{\left(a_{i}, b_{i}\right)\right\}_{i=1, \ldots m}$, their corresponding probabilities can be found by solving Eq. (9) with $x=a_{1}, \ldots, a_{m-1}$ written in the form (for $n=2$ )

$$
\left(\begin{array}{cccc}
1-\frac{a_{1}}{a_{2}} & 1-\frac{a_{1}}{a_{3}} & \cdots & 1-\frac{a_{1}}{a_{m}} \\
0 & 1-\frac{a_{2}}{a_{3}} & \cdots & 1-\frac{a_{2}}{a_{m}} \\
\vdots & \vdots & \ddots & \vdots \\
0 & 0 & \cdots & 1-\frac{a_{m-1}}{a_{m}}
\end{array}\right)\left(\begin{array}{c}
p_{2} \\
p_{3} \\
\vdots \\
p_{m}
\end{array}\right)=\left(\begin{array}{c}
b_{1} \\
b_{2} \\
\vdots \\
b_{m-1}
\end{array}\right)
$$

The solution is $p_{1}=1-\left(p_{2}+\cdots+p_{m}\right)$ and

$$
p_{i}=\frac{a_{i}\left[b_{i-1}\left(a_{i+1}-a_{i}\right)-b_{i}\left(a_{i+1}-a_{i-1}+b_{i+1}\left(a_{i}-a_{i-1}\right)\right)\right]}{\left(a_{i+1}-a_{i}\right)\left(a_{i}-a_{i-1}\right)} \quad \text { for } \quad i=2, \ldots m \text {, }
$$

with auxiliary point $\left(a_{m+1}, b_{m+1}\right)$, where $a_{m+1} \geq a_{m}$ and thus $b_{m+1}=0$.

ExAmple 8 . Let $F(x)=\min \left(1, x^{p}\right)$ be the parametric family from the Example 4, then Figure 7 shows contour plots and samples from copulas generated by (9) with $a_{i}=F\left(u_{i}\right)$, where $u_{i}$ is sampled from uniform distribution $\mathrm{U}(0,1)$, and $p\left(a_{i}\right)=F\left(a_{i}\right)-F\left(a_{i-1}\right)$, where $i=1, \ldots m$, and $a_{0}=0$.

In the following examples we exercise pointwise convergence and show a piecewise linear copula corresponding to the simplest non-trivial case $n=m=2$.

EXAMPle 9. For the simplest case, $n=2, a_{i}=\frac{i}{m}$ and $p\left(a_{i}\right)=\frac{1}{m}, i=1, \ldots m$ (evenly spaced and uniformly distributed), we get

$$
f_{m}^{(-1)}(x)=\sum_{i=1}^{m} \frac{1}{m} \max \left(0,1-\frac{m x}{i}\right) .
$$

If $f_{m}^{(-1)}(x)$ is to converge to $f^{(-1)}(x)=1-x+x \log x$ for $x<1$ and 0 elsewhere, it needs to converge in any point $x \in] 0,1[$. Let us examine the convergence, say, in $x=\frac{1}{2}$, where

$$
\begin{aligned}
f_{m}^{(-1)}\left(\frac{1}{2}\right) & =\frac{1}{m} \sum_{i=1}^{m} \max \left(0,1-\frac{m \frac{1}{2}}{i}\right) \\
& =\frac{1}{m} \sum_{i=\left\lfloor\frac{m}{2}\right\rfloor+1}^{m}\left(1-\frac{m}{2 i}\right) \\
& =\frac{1}{m} \sum_{i=1}^{\frac{m}{2}} \frac{i}{i+\frac{m}{2}} \\
& =\frac{1}{m} \sum_{i=1}^{\frac{m}{2}}\left(1-\frac{\frac{m}{2}}{i+\frac{m}{2}}\right) \\
& =\frac{1}{2}-\frac{1}{2} \sum_{i=\left\lfloor\frac{m}{2}\right\rfloor+1}^{m} \frac{1}{i} .
\end{aligned}
$$




\section{CONVERGENCE OF LINEAR APPROXIMATION OF ARCHIMEDEAN GENERATOR}
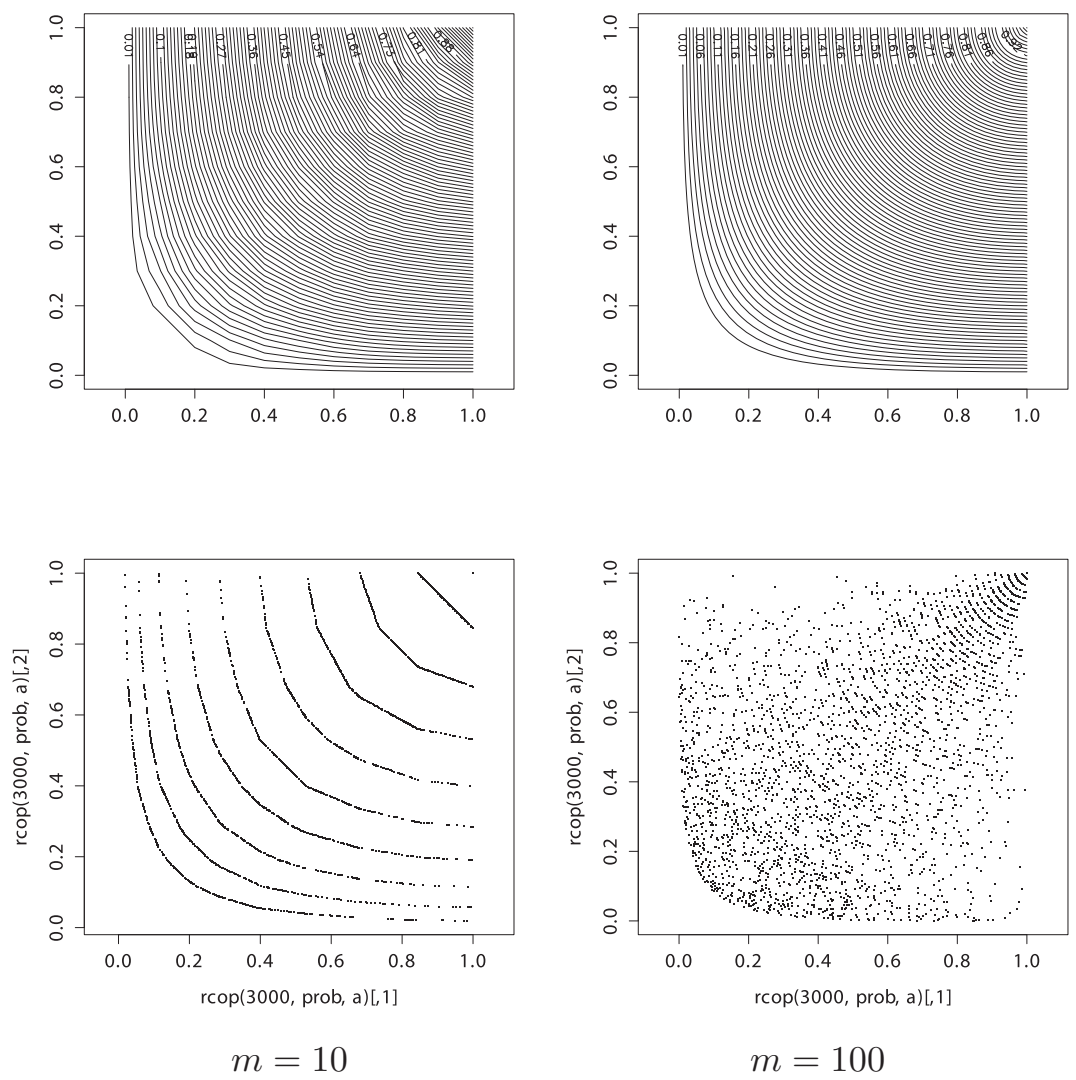

Figure 7. Sampling from approximation of copula family constructed in Example 4 with $p=0.5$.

Then indeed

$$
\lim _{m \rightarrow \infty} f_{m}^{(-1)}\left(\frac{1}{2}\right)=\frac{1}{2}-\frac{1}{2} \int_{\frac{m}{2}}^{m} \frac{1}{x} d x=\frac{1}{2}-\frac{1}{2}[\ln x]_{\frac{m}{2}}^{m}=\frac{1}{2}-\frac{1}{2} \ln 2=f^{(-1)}\left(\frac{1}{2}\right) .
$$

ExAmple 10. Following Example 9, it might help to picture the approximation copula on a simple setting. Due to Example 2 we already know that the trivial case $m=1$ leads to the weakest copula $W$. With $m=2$ we get

$$
F_{2}(x)= \begin{cases}0, & x<\frac{1}{2} \\ \frac{1}{2}, & \frac{1}{2} \leq x<1 \\ 1, & 1 \leq x\end{cases}
$$


thus

$$
f_{2}^{(-1)}(x)=\left\{\begin{array}{ll}
1-\frac{3}{2} x, & x<\frac{1}{2}, \\
\frac{1}{2}-\frac{1}{2} x, & \frac{1}{2} \leq x<1, \\
0, & 1 \leq x
\end{array} \quad \text { and } \quad f_{2}(x)= \begin{cases}1-2 x, & 0 \leq x \leq \frac{1}{4} \\
\frac{2}{3}(1-x), & \frac{1}{4}<x \leq 1\end{cases}\right.
$$

shown in Figure 8 a), which leads to copula $C_{2}$ expressed in Figure 8 b).

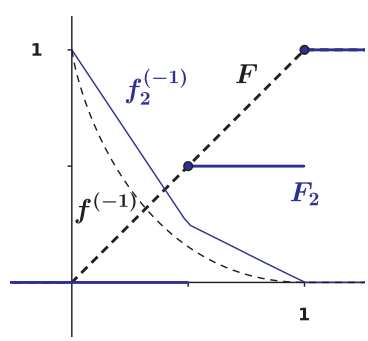

a)

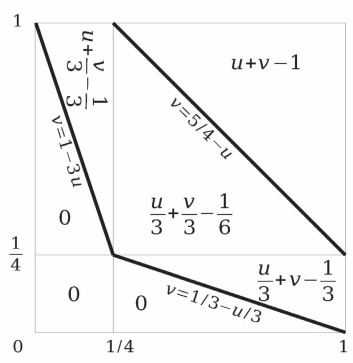

b)

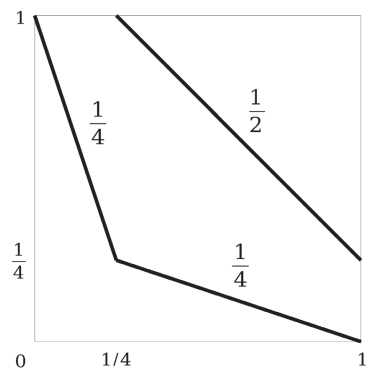

c)

Figure 8. a) Distance function, generator (inverse) and b) copula, that correspond to uniform distribution approximated in $m=2$ equally spaced points. c) Probability mass concentrated on copula support.

To compute measures of dependence (concordance) such as Spearman's rho and Kendall's tau corresponding to singular copula it is generally a challenge, yet for this simple settings it might be an interesting exercise. Since the copula $C_{2}$ is piecewise linear, the whole probability mass is concentrated on its support, thus to evaluate the expected values (especially in (44)) one need to find out distribution of the probability. In our case, it is depicted in Figure $8 \mathrm{c}$ ). By expressing variable $v$ in terms of $u$ the double integral reduces to one-dimensional integral, then

$$
E[U V]=2 \int_{0}^{1 / 4} u(1-3 u) \frac{\frac{1}{4}}{\frac{1}{4}} d u+\int_{1 / 4}^{1} u\left(\frac{5}{4}-u\right) \frac{\frac{1}{2}}{\frac{3}{4}} d u=\frac{2}{64}+\frac{11}{64}=-\frac{13}{64}
$$

and

$$
\begin{aligned}
E[C(U, V)]= & 2 \int_{0}^{1 / 4} \max \left(0, u+\frac{1-3 u-1}{3}\right) \frac{\frac{1}{4}}{\frac{1}{4}} d u \\
& +\int_{1 / 4}^{1} \max \left(\frac{1}{3}\left(u+\frac{5}{4}-u-\frac{1}{2}\right), u+\frac{5}{4}-u-1\right) \frac{\frac{1}{2}}{\frac{3}{4}} d u=0+\frac{1}{8}
\end{aligned}
$$


thus $\rho_{2}=12 \frac{13}{64}-3=-\frac{9}{16}$ and $\tau_{2}=4 \frac{1}{8}-1=-\frac{1}{2}$, where the subscript 2 conforms the notation of generator. Although we cannot find explicit form of the original generator $f$ (that corresponds to uniform distribution $\mathrm{U}[0,1]$ ) and analytically calculate $\rho$, we still can get $\tau=1-\int_{0}^{1} t\left((1-t+x \ln t)^{\prime}\right)^{2} d t=1-4 \int_{0}^{1} t \ln ^{2} t d t=0$ to measure accuracy of our $m=2$ approximation.

\section{Conclusion}

We have discussed a new construction method for obtaining additive generators proposed by McNeil and Nešlehová [8], the so-called Williamson n-transform, and illustrated it by some examples. Some of the generators were shown to not have an explicit form due to non-invertability. Thus a natural approach to utilize any such parametric family is to approximate it by piecewise (in $2 \mathrm{D}$ case) linear functions with sufficiently dense breakpoints. We showed that due to the equivalence of convergences of positive distance functions, fixed additive generators and copulas, we may approximate any $n$-dimensional Archimedean copula by a transformation of convex sum of Dirac functions (though feasible mainly in 2D). We showed some simple examples, including calculation of correlation coefficients related to a singular copula.

\section{REFERENCES}

[1] BACIGÁL, T.-JURÁŇOVÁ, M.-MESIAR, R.: On some new constructions of Archimedean copulas and applications to fitting problems, Neural Netw. World 20 (2010), 81-90.

[2] BACIGÁL, T.-MESIAR, R.-NAJJARI, V.: Generators of copulas and aggregation, Inform. Sci. (submitted).

[3] CHARPENTIER, A.-SEGERS, J.: Convergence of Archimedean copulas, Statist. Probab. Lett. 78 (2008), 412-419.

[4] JÁGR, V.-KOMORNÍKOVÁ, M.-MESIAR, R.: Conditioning stable copulas, Neural Netw. World 20 (2010), 69-79.

[5] JOE, H.: Multivariate Models and Dependence Concepts. Chapman and Hall, London, 1997.

[6] KLEMENT, E. P.-MESIAR, R.-PAP, E.: Triangular norms. Kluwer Acad. Publ., Dodrecht, 2000.

[7] KLEMENT, E. P.-MESIAR, R.-PAP, E.: Transformations of copulas, Kybernetika 41 (2005), 425-434.

[8] MCNEIL, A. J.-NEŠLEHOVÁ, J.: Multivariate Archimedean copulas, d-monotone functions and $l_{1}$-norm symmetric distributions, Ann. Statist. 37 (2009), 3059-3097.

[9] MENGER, K.: Statistical metrics, Proc. Natl. Acad. Sci. USA 28(12) (1942), 535-537.

[10] MESIAR, R.: On the pointwise convergence of continuous Archimedean t-norms and the convergence of their generators, BUSEFAL $\mathbf{7 5}$ (1998), 39-45. 


\section{TOMÁŠ BACIGÁL — MÁRIA ŽDÍMALOVÁ}

[11] MICHIELS, F.-DE SCHEPPER, A.: How to improve the fit of Archimedean copulas by means of transforms, Statist. Papers 53 (2012), 345-355.

[12] MOYNIHAN, R.: On $\tau_{T}$ semigroups of probability distributions II, Aequationes Math. 17 (1978), 19-40.

[13] NELSEN, R. B.: An Introduction to Copulas. Springer, New York, 2006.

[14] RÉNYI, A.: Wahrscheinlichketsrechnung mit einem Anhang über Informationstheorie. Deutsche Verlag der Wissenschaften, Berlin, 1962.

[15] SCHWEIZER, B.-SKLAR, A.: Probabilistic metric spaces. Courier Dover Publ., 1983.

[16] SKLAR, A.: Fonctions de répartition à $n$ dimensions et leurs marges, Publ. Inst. Statist. Univ. Paris 8 (1959), 229-231.

[17] STUPŇAnOVÁ, A.-KOLESÁROVÁ, A.: Associative $n$-dimensional copulas, Kybernetika 47 (2011), 93-99.

[18] WILLIAMSON, R. E.: Multiply monotone functions and their Laplace transforms, Duke Math. J. 23 (1956), 189-207.

Received April 13, 2017

Faculty of Civil Engineering Slovak University of Technology in Bratislava

Radlinského 11

SK-810-05 Bratislava

SLOVAKIA

E-mail: bacigal@math.sk zdimalova@math.sk 